\title{
Vibrations and health effects at sea
}

\author{
Anker Jensen ${ }^{1}$, Jørgen Riis Jepsen ${ }^{1,2}$ \\ ${ }^{1}$ Department of Occupational Medicine, Hospital of South Western Jutland, Esbjerg, Denmark \\ ${ }^{2}$ Centre of Maritime Health and Society, Institute of Public Health, University of Southern Denmark, Esbjerg, Denmark
}

Our recent review [1] of the current international literature on vibrations and health effects in a maritime context was based on a lecture at the recent NIVA-course on Maritime Occupational Medicine in Helsingør in May this year. The review was limited to research results that were published in English and dealing with the long-term effects of vibrations. Consequently, we may have missed research on specific additional aspects that somebody could regard as of relevance.

Few measurements of vibrations on board ships have been published. The levels in various workplaces and living quarters on board a roll-on/roll-off vessel studied by Szczepanski [2] were found acceptable according to Polish standards. The prevailing frequency was reported to be $8 \mathrm{~Hz}$.

Whole body vibration may influence the hypothalamic-pituitary-adrenal axis and consequently the vegetative neuroregulation and the behavioural reactions of seafarers. Through these mechanisms, whole body vibration may contribute to outcomes such as fatigue and seasickness, which, however, was out of the scope of our review, because they were covered by other lectures at the course.

Vibrations at sea are a complex phenomenon caused by mechanical equipment on board, such as the main shaft, but may also occur consequent to the propulsion of ships, the impact of waves and the rocking of the ship. Therefore, many elements interact such as the equipment on board, elements of design, speed, and the weather and sea. However, while the accelerations caused by these factors per se may cause vibration, the adverse health impacts of largescale acceleration should rather be assigned to the high levels of mechanical energy being transmitted to human tissue from this source than from the vibration that it may cause secondarily.

For that reason we have not covered issues such as stress fractures, which is a rare event in a maritime context but may follow repeated physical stress from heavy impacts deriving from the ship's speed and acceleration, and from the simultaneous impact on the ship of waves, e.g. in fast torpedo vessels. Cavitation of inner organs is another rare and related health outcome. Both have been associated with high-speed pressure waves (blast), but in a maritime context, however, we do regard these as acute adverse exposures from excessive accelerations rather than from vibrations. Both are interesting issues but we deliberately aimed to cover vibrations in a classical way (long term effects) and consequently excluded such more direct and acute exposures and their effects.

We certainly would appreciate comments from other researchers dealing with vibrations at sea.

\section{REFERENCES}

1. Jensen A, Jepsen JR. Vibration on board and health effects. Int Marit Health 2014; 64: 58-60.

2. Szczepanski C. Evaluation of the exposure of a merchant ship crew to mechanical vibration during a sea voyage. Bull Mar Trop Med Gdynia 1990; 41: 1-4. 